\title{
Development and evaluation of poly(dimethylsiloxane) based composite coatings for icephobic applications
}

Junpeng Liu ${ }^{1}$, Jie Wang ${ }^{1}$, Luca Mazzola ${ }^{2}$, Halar Memon ${ }^{1}$, Tamal Barman ${ }^{1}$, Barbara Turnbull ${ }^{1}$, Mingione Giuseppe ${ }^{2}$, Kwing-So Choi ${ }^{1}$ and Xianghui Hou ${ }^{1 *}$

${ }^{1}$ Faculty of Engineering, University of Nottingham, University Park, Nottingham NG7 2RD, $\mathrm{UK}$;

${ }^{2}$ CIRA, Italian Aerospace Research Center, Innovative Materials Lab, Via Maiorise 1, 81043 Capua (CE), ITALY

* Correspondence: xianghui.hou@nottingham.ac.uk;

\begin{abstract}
:
Formation and accretion of ice on the leading edge surface of aircrafts wings may lead to disasters. The current de-icing system for aircraft will build up weight, increase energy consumption and add complexity to the aircraft systems. Development of icephobic coatings is a potential solution to prevent ice formation and/or reduce accretion on the critical surface of aircraft. Icephobic coatings based on poly(dimethylsiloxane) (PDMS) with modification by fluorosilane and incorporation of silica nanoparticles have been fabricated. The hydrophobicity of the coatings has been measured in normal conditions with atmospheric pressure and room temperature, showing improvement of hydrophobicity by the fluorination of PDMS and incorporation of silica nanoparticles. The water droplet icing behaviour shows better anti-icing performance for fluorinated PDMS (F-PDMS)/silica coatings with a rough surface. The ice adhesion strength test results show that F-PDMS coatings without silica nanoparticles have lower ice adhesion strength implying better de-icing performance. The wettability of the coatings was also measured at reduced pressure and temperature, to study the mechanism of higher ice adhesion strength of F-PDMS/silica coatings comparing with F-PDMS based coatings. For the design and fabrication of icephobic coatings, compromise on the roughness
\end{abstract}


induced hydrophobicity may become a critical requirement to avoid mechanical interlock between the ice and the rough surface.

Keywords: Icephobicity, Poly (dimethylsiloxane), Wettability, Ice adhesion strength, Water droplet erosion

\section{Introduction}

Ice formation and accumulation on critical aircraft surfaces such as leading edges of wings is a serious hazard that will disturb smooth air flow and greatly degrade the ability to generate lift and adversely affect the handling qualities of the aircraft. An investigation of icing-related accidents and incidents sponsored by the U. S. Department of Transportation reported 308 in-flight icing events including 166 classified as accidents [1]. When aircraft flight through supercooled water droplets which often present in stratiform and cumulus clouds, ice may form and start to accumulate. Therefore, the ice protection during flight is a critical issue for flight safety [2]. An in-flight de-icing method on existing aircraft is to heat the critical components using hot air bled from the engine compressor [3] and/or use electrothermal heating. However, the in-flight de-icing system will build up weight, increase fuel consumption and add complexity to the aircraft systems.

Aiming to improve the energy efficiency and operational effectiveness of the current antiicing or de-icing system, especially during in-flight, in an environmentally friendly way, applying icephobic coatings with anti-icing function, low ice adhesion and mechanical durability on the critical regions of aircraft is a potential solution. Although it is an argument that some superhydrophobic surfaces may not necessarily be icephobic, more researchers suggested surface-roughness-induced hydrophobicity can be used to design icephobic coatings $[4-17]$. 
The hydrophobicity is normally evaluated in atmospheric pressure and room temperature. However, during flight, the highest probability to have icing formation and accumulation is between the altitude of 4300 meters and 5000 meters with a temperature of $-15 \sim-12{ }^{\circ} \mathrm{C}$ and pressure of about 0.5 Bar $[18,19]$. In the flight condition, the surface-roughness-induced hydrophobicity may act differently as it under normal conditions. Gradually, it has been realized that the water contact angle should be measured at low temperatures when correlating the water contact angle with icephobic performance [20]. However, attention has not been paid properly to the possible influence of the environmental pressure. Therefore, hydrophobicity study in reduced pressure and/or temperature is essential to evaluate the correlation between hydrophobicity and icephobicity.

Poly(dimethylsiloxane) (PDMS) is a good candidate for icephobic coatings due to its low surface energy [21] and was explored for anti-icing applications [22, 23]. However, it is unlikely to reach water contact angle of above $120^{\circ}$ using smooth PDMS. To enhance the hydrophobicity and icephobicity, PDMS was further modified using fluorosilane and incorporated with silica nanoparticles to lower down the surface energy and increase the capability of repelling water. Icephobic evaluation of the coatings including water droplet icing behaviour and ice adhesion strength were carried out. Moreover, the wettability of coatings based on fluorinated PDMS (F-PDMS) with silica nanoparticles (F-PDMS/silica) was also evaluated under reduced pressure and/or reduced temperature, to investigate the variation in hydrophobicity/icephobicity and mechanisms involved.

\section{Experimental details}

\subsection{Fabrication of PDMS and PDMS/silica coatings}


Poly(dimethylsiloxane) hydroxyl terminated (PDMS) and methanol were purchased from Sigma-Aldrich Company (Dorset, UK). Fumed silica nanoparticles with average size of $15 \mathrm{~nm}$ were purchased from Evonik. Xylene was purchased from Fisher Scientific Company. (Heptadecafluoro-1,1,2,2-tetrahydrodecyl)trimethoxysilane was purchased from Fluorochem. All chemicals were used as received. The PDMS was fluorinated by mixing with (Heptadecafluoro-1,1,2,2-tetrahydrodecyl)trimethoxysilane (weight percentage of $6.8 \%$ ) in xylene and kept stirring for 2 hours. Then the F-PDMS was mixed with silica (30 wt $\%$ and 40 $w t \%)$ in xylene and stirred for 2 hours. The F-PDMS and F-PDMS/silica were used to coat the sand-blasted aluminium alloy (2024-T4) substrates by brushing. Then the samples were transferred to a furnace for heat treatment at $150{ }^{\circ} \mathrm{C}$ for $150 \mathrm{mins}$ to remove the organic solvent and complete the curing. For comparison, the pristine PDMS were also used to prepare coatings.

\subsection{Characterization of structure and morphology}

Fourier transform infrared (FTIR) spectra were recorded by a spectrometer (Spectrum One, Perkin Elmer, Akron, OH, USA) using attenuated total reflection mode. The surface topography and roughness were investigated by an Optical Profiler (Zeta - 20) and Atomic Force Microscopy (AFM, Bruker, FastScan) using PeakForce Mapping mode. The surface morphology of the coatings before and after erosion test was measured by the Scanning Electron Microscope (SEM, 6490LV, JOEL) under an acceleration voltage of $10 \mathrm{kV}$ after Pt was deposited on the samples to prevent charging by the electron beam.

\subsection{Test of hydrophobicity and icephobicity}

Static water contact angle (CA), advancing CA, receding CA and CA hysteresis of the surfaces under normal conditions with atmospheric pressure and room temperature were characterized using a contact angle goniometer (FTA200, First Ten Angstroms, Inc., Portsmouth, VA, USA) with pumping out rate of $1 \mu \mathrm{L} / \mathrm{s}$. Besides, the water contact angles 
were also measured under simulated flight conditions under 0.5 bar pressure and/or temperature of $-12{ }^{\circ} \mathrm{C}$ following the method described elsewhere [24].

The water droplet icing tests were performed by monitoring the water droplets with a volume of $4 \mu \mathrm{L}$ on five spots of coated samples and uncoated aluminium substrates for comparison on a cold plate setting at $-10{ }^{\circ} \mathrm{C}[25]$. The average icing duration was recorded to evaluate the antiicing performance.

Ice adhesion tests were performed using a centrifuge method with a glaze ice block (mass of $1.3 \mathrm{~g}$ ) in a chamber with a temperature of $-5^{\circ} \mathrm{C}$. Using the rotation speed at the detachment of the glaze ice block, the ice adhesion strength is calculated using the ice block mass and beam length [26]:

$$
F=m r \omega^{2}
$$

Where $F$ is the centrifugal force $(\mathrm{N}), m$ is the mass of ice block $(\mathrm{kg}), r$ is the radius of the beam ( $m$ ) and $\omega$ is the speed of rotation $(\mathrm{rad} / \mathrm{s})$. From the centrifugal force, the shear stress is determined:

$$
\tau=\frac{F}{A}
$$

Where $A$ is the Area iced $\left(\mathrm{m}^{2}\right), \tau$ is the shear stress $(\mathrm{Pa})$.

\subsection{Water impinging erosion test}

To evaluate the coating durability, erosion test (as shown in Figure 1) was carried out. During the test, water droplets under compressed gas were sprayed onto the coated specimen from the nozzle connected with the water tank using the following parameters including gas pressure of $103.4 \mathrm{kPa}$, velocity of up to $37 \mathrm{~m} / \mathrm{s}$, liquid flow rate of $72 \mathrm{~mL} / \mathrm{min}$, distance between the nozzle and the specimen of $4 \mathrm{~cm}$ and duration of $90 \mathrm{~min}$. The water contact angles and surface morphology of the coatings were measured before and after the erosion test. 


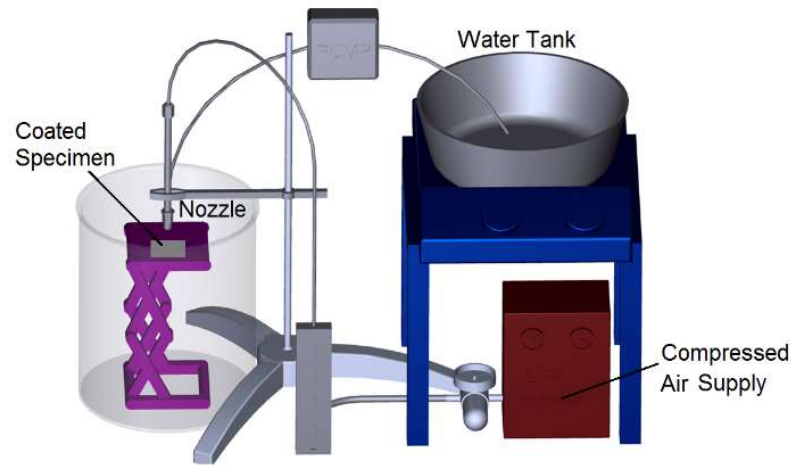

Figure 1 Schematic diagram of the water impinging erosion test.

\section{Results and discussion}

\subsection{Fluorination of PDMS}

In general, the interaction between the substrate and ice includes mechanical adhesion and electrostatic force. Fluoropolymer can reduce the electrostatic force between the substrate and ice due to the low dielectric constant [27]. For a heterogeneous polymer surface containing both fluorocarbon and siloxane, the interaction energies between the surface and water droplet would be decreased. Therefore, in principle, modification of siloxane by fluorocarbon would contribute to better hydrophobicity and icephobicity [21].

FTIR spectra of the pristine PDMS and F-PDMS modified using fluorosilane were collected to investigate the composition and structure change during the fluorination. Both PDMS and fluorosilane contain $\mathrm{CH}_{3}$ groups and the unique groups for fluorosilane are the $\mathrm{CF}_{2}$ and $\mathrm{CH}_{2}$ groups. It can be seen from the FTIR spectra shown in Figure 2 after fluorination two new peaks centred at $1730 \mathrm{~cm}^{-1}$ and $2850 \mathrm{~cm}^{-1} / 2916 \mathrm{~cm}^{-1}$ appear which can be assigned to vibration mode of $\mathrm{CF}_{2}$ and $\mathrm{CH}_{2}$ indicating existing of fluorosilane composition [28]. Besides, the peak at $2963 \mathrm{~cm}^{-1}$ is found on the original PDMS and modified PDMS and it can be attributed to the $\mathrm{CH}_{3}$ stretching vibrations originating from the methyl side groups of PDMS and fluorosilane which are an overlap of the absorption area [29]. The fluorination of PDMS 
using fluorocarbon will create a surface with better hydrophobicity by reducing the surface energy instead of increasing the roughness.

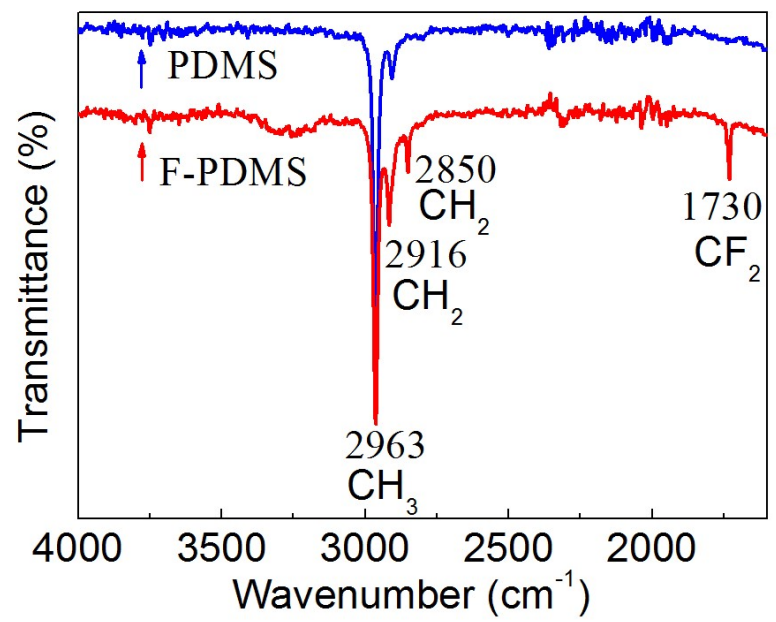

Figure 2 FTIR spectra of pristine PDMS and F-PDMS modified using fluorosilane.

\subsection{Hydrophobicity and surface morphology}

The hydrophobicity for the samples with pristine PDMS, F-PDMS and F-PDMS/silica was evaluated using water contact angle measurement. Figure 3 shows the water contact angles of $105^{\circ}$ for the pristine PDMS coatings, $124^{\circ}$ on F-PDMS based coating, $132^{\circ}$ on F-PDMS/(30 $\mathrm{wt} \%$ )silica and $157^{\circ}$ on F-PDMS/(40 wt\%)silica coatings, indicating an improvement of hydrophobicity by the fluorination of PDMS and the incorporation of silica nanoparticles. The improvement of hydrophobicity by fluorination of PDMS can be attributed to the lower surface energy due to the nature of fluorosilane. The hydrophobicity improvement by addition of silica nanoparticles is likely to be attributed to the change of surface morphology.
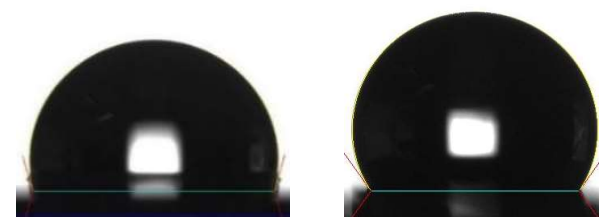

(a) $\mathrm{WCA}=105^{\circ}$

(b) $\mathrm{WCA}=124^{\circ}$

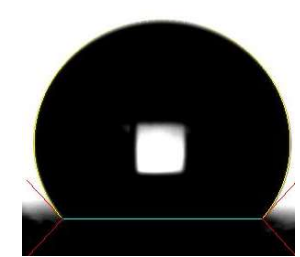

(c) $\mathrm{WCA}=132^{\circ}$

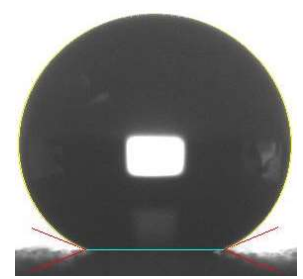

(d) $\mathrm{WCA}=157^{\circ}$ 
Figure 3 Typical water contact angles of (a) pristine PDMS coatings, (b) F-PDMS based coating, (c) F-PDMS/(30 wt\%) silica nanoparticle and (d) F-PDMS/(40 wt\%) silica nanoparticle coating.

The advancing contact angle, receding angle and contact angle hysteresis have been widely used to distinguish different modes or regimes of wetting including Wenzel, Cassie-Baxter, lotus and petal, and a transition mode between them [30]. Rose petal tends to have large CA hysteresis and high water adhesion as opposed to lotus leaf with low CA hysteresis and low adhesion [30]. Table 1 shows the advancing CA, receding CA and CA hysteresis of pristine PDMS, F-PDMS and F-PDMS/silica coatings. It can be seen that CA hysteresis reduced from $23^{\circ}$ to $16^{\circ}$ and $2^{\circ}$ after fluorination and incorporation of silica nanoparticles, indicating lower water adhesion. Besides, smaller CA hysteresis is normally found on the surface with CassieBaxter state due to the small solid-liquid contact area and is expected to allow water droplet to roll off easily [30, 31]. Meanwhile, larger CA hysteresis is normally found on the surface with Wenzel state and it is more difficult to roll off the water droplets as water follows the contours of the rough surface [32]. Therefore, reduction of CA hysteresis may benefit the anti-icing function of the coatings by allowing water rolling off from the surface.

Table 1 Advancing contact angle, receding contact angle and contact angle hysteresis of pristine PDMS, fluorinated PDMS and fluorinated PDMS with silica nanoparticles coatings.

\begin{tabular}{|l|l|l|l|}
\hline & $\begin{array}{l}\text { Advancing CA } \\
\left({ }^{\circ}\right)\end{array}$ & $\begin{array}{l}\text { Receding CA } \\
\left({ }^{\circ}\right)\end{array}$ & $\begin{array}{l}\text { CA hysteresis } \\
\left({ }^{\circ}\right)\end{array}$ \\
\hline Pristine PDMS coating & $126 \pm 1.7$ & $103 \pm 1.5$ & $23 \pm 2.5$ \\
\hline F-PDMS based coating & $123 \pm 4.4$ & $107 \pm 0.13$ & $16 \pm 4.5$ \\
\hline F-PDMS/silica coating & $134 \pm 0.43$ & $132 \pm 0.29$ & $2 \pm 0.6$ \\
\hline
\end{tabular}


Based on the known chemistries, the highest contact angle on a purely smooth surface is $\sim 120^{\circ}$ [33]. To achieve significantly reduced ice adhesion with a correlation of water contact angle, micro and nanoscale roughness were needed in principle [33] which is the reason to incorporate fumed silica nanoparticles. The surface topography and roughness of F-PDMS coating and F-PDMS/silica coatings were investigated by Optical Profiler and AFM and the results are shown in Figure 4. The Roughness Average (Ra) measured in the range of $450 \mu \mathrm{m}$ $\times 550 \mu \mathrm{m}$ by Optical Profiler are $1.6 \mu \mathrm{m}$ and $7.3 \mu \mathrm{m}$ for F-PDMS and F-PDMS/silica respectively. The Ra measured in the range of $1 \mu \mathrm{m} \times 1 \mu \mathrm{m}$ are $2.0 \mathrm{~nm}$ and $56.4 \mathrm{~nm}$ by AFM for F-PDMS and F-PDMS/silica respectively. The significantly increased roughness and the images show the F-PDMS coatings are relatively smooth, while the F-PDMS/silica coatings show a rough surface which increases surface area and creates significant irregularities. Air pockets can be formed at the solid/liquid interface when contacting with water droplets, which is favourable to obtaining higher water repelling capability. The increased roughness and reduced CA hysteresis by the incorporation of silica nanoparticles suggest a transition from the Wenzel regime of surface wetting to the Cassie-Baxter wetting [34] which will benefit the antiicing performance.

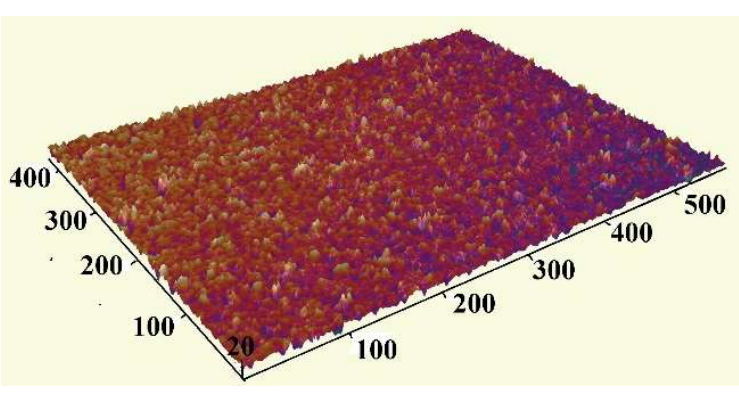

(a)

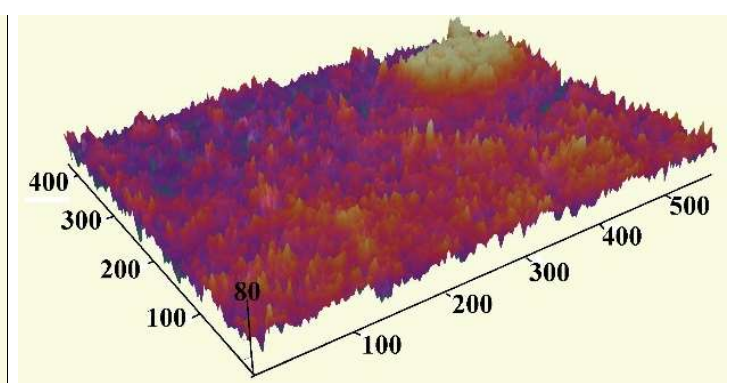

(b) 


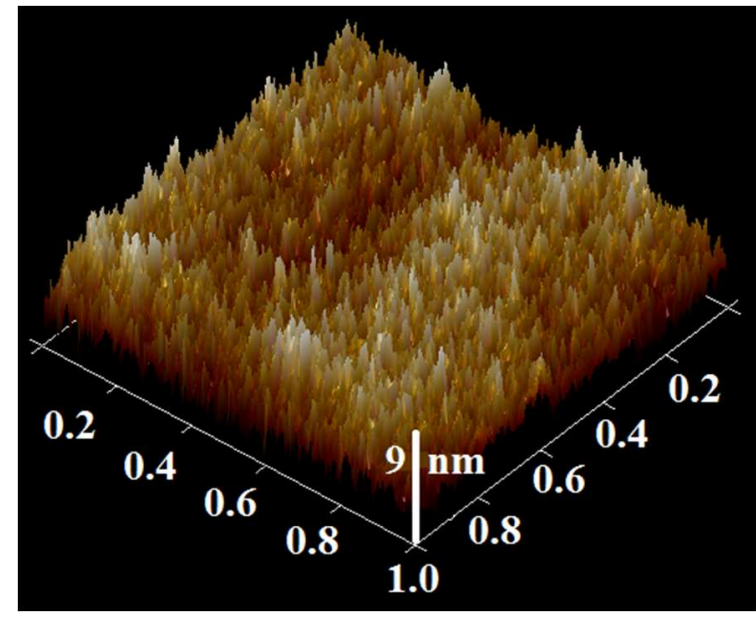

(c)

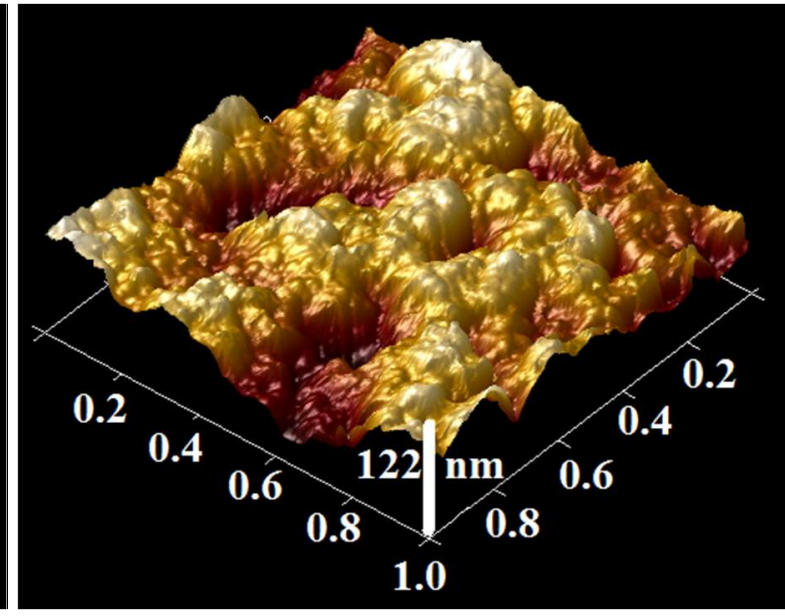

(d)

Figure 4 3D optical images of F-PDMS coatings (a) and F-PDMS/silica coating (b) with scanning area of $450 \mu \mathrm{m} \times 550 \mu \mathrm{m}$ and scale bar in $\mu \mathrm{m}$; AFM images of F-PDMS coatings (c) and F-PDMS/silica coating (d) with scanning area of $1 \mu \mathrm{m}$ and scale bar in $\mathrm{nm}$.

\subsection{Anti-icing and de-icing performance}

It was believed that the ice nucleation rate and macroscopical growth velocity can be greatly reduced by a hydrophobic surface owing to low thermal transfer rate caused by a low actual solid-liquid contact area between the water droplets and the hydrophobic surface according to classical nucleation theory and observation [35]. To evaluate anti-icing performance of the coatings, water droplet icing duration on the coating surface was measured. Figure 5 shows the average duration for water droplets to frozen is 3.2 seconds, 9.8 seconds, 11.2 seconds and 13.8 seconds for the aluminium substrate, coatings based on pristine PDMS, F-PDMS and FPDMS/silica nanoparticles, respectively, indicating that better anti-icing performance is correlated with better hydrophobicity. The thermal transfer rate is lower if the solid-liquid contact area is smaller due to high water contact angle. 


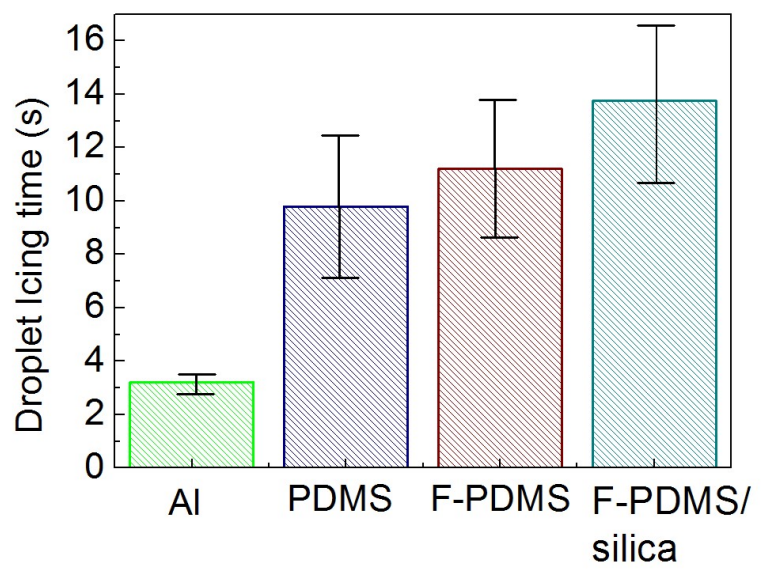

Figure 5 Water droplet icing test results for the aluminium substrate, pristine PDMS, F-PDMS and F-PDMS/silica coatings.

Besides anti-icing performance, ice adhesion strength is another important parameter to evaluate the icephobicity which can reflect the degree of difficulty during de-icing process. For coatings with low ice adhesion strength, the ice can be removed with a less mechanical force which is desirable for energy efficiency during de-icing process. It was revealed that the ice adhesion strength is linearly correlated with $1+\cos \theta_{e}$, with $\theta_{e}$ standing for the estimated equilibrium contact angle which implies that a low ice adhesion strength can be obtained from hydrophobic surfaces [5]. The ice adhesion strengths of F-PDMS and F-PDMS/silica coatings were measured using the centrifuge method. As observed in Figure 6, the shear stresses between the ice and F-PDMS or F-PDMS/silica coatings are all lower than that of the untreated aluminium substrates and are well below $100 \mathrm{kPa}$ which is considered as the threshold for icephobicity showing icephobicity [36]. However, the ice adhesion of F-PDMS/silica coatings is higher than F-PDMS coatings which is not expected according to the conventional understanding of the correlation between the hydrophobicity and icephobicity. Considering the de-icing purpose, the F-PDMS coatings show better performance comparing with FPDMS/silica coatings. It is easy to imagine that the rough surface of F-PDMS/silica may form mechanical interlocks between the ice and surface which causes higher ice adhesion. To 
investigate the underlying mechanism, contact angles on F-PDMS/silica coatings under conditions with reduced temperature and/or reduced pressure were measured.

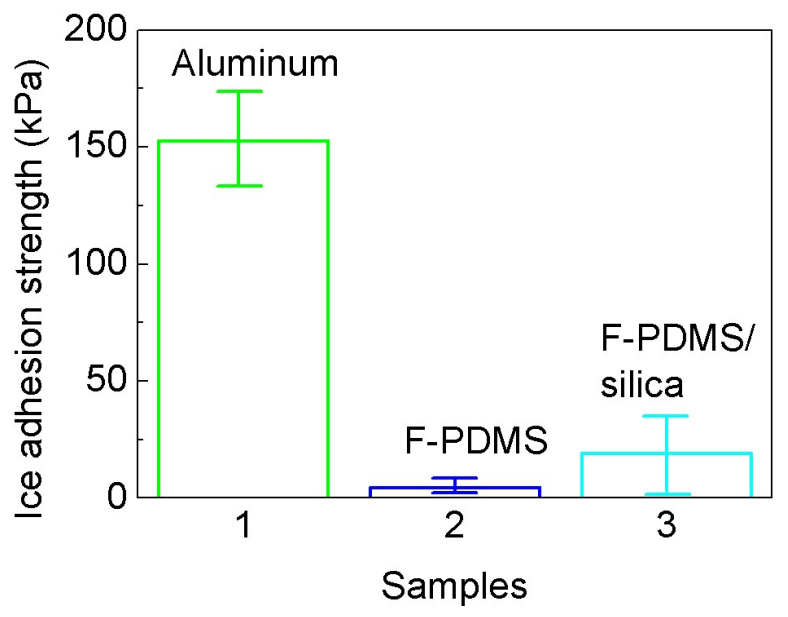

Figure 6 Ice adhesion strengths of coatings based on F-PDMS and F-PDMS/silica on Al substrates and untreated bare aluminium substrates.

\subsection{Wetting behaviours at reduced pressure and temperature}

Conventionally, superhydrophobic surface with high roughness and/or low surface energy layer was widely explored to be used for icephobic applications [37]. In general, the hydrophobicity was measured in normal conditions with atmospheric pressure and room temperature. Aiming for applications as ice protection for aircraft, hydrophobicity / icephobicity in-flight conditions with reduced pressure and temperature is helpful to evaluate the suitability. The highest possibility of icing formation for an aircraft occurs at the altitude of 4300 to 5000 meters with the pressure of 0.5 bar and temperature of $-12{ }^{\circ} \mathrm{C}[18,19]$. Figure 7 shows the water contact angle of F-PDMS/silica coatings of $119^{\circ}, 118^{\circ}$ and $104^{\circ}$ measured at reduced pressure $\left(\mathrm{P}=0.5 \mathrm{Bar}, \mathrm{T}=24^{\circ} \mathrm{C}\right)$, reduced temperature $\left(\mathrm{P}=1 \mathrm{bar}, \mathrm{T}=-12{ }^{\circ} \mathrm{C}\right)$ and simulated flight conditions with a reduced pressure and temperature $\left(\mathrm{P}=0.5 \mathrm{Bar}, \mathrm{T}=-12^{\circ} \mathrm{C}\right)$. It is obvious that the water contact angle measured at reduced pressure and/or temperature is much lower than the water contact angle of $157^{\circ}$ measured at atmospheric pressure and room temperature. The reasons can be explained by the less functionality of air pockets on the 
roughness-induced hydrophobic surface of F-PDMS/silica coatings and higher surface free energy under reduced pressure and/or temperature [38]. The results can partially explain the higher ice adhesion strength of F-PDMS/silica coating to be stronger chemical bonds between water and surface under reduced temperature [38].

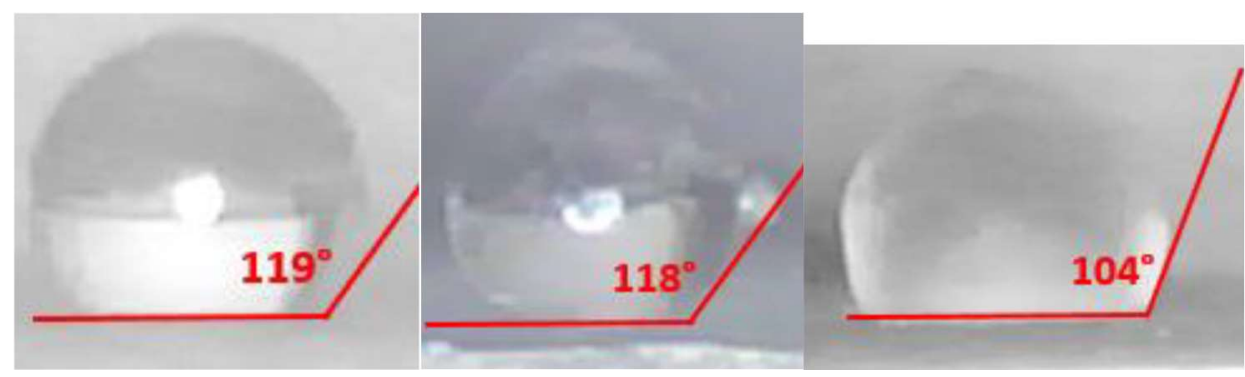

(a)

(b)

(c)

Figure 7 Typical water contact angles of F-PDMS/silica based coatings measured at different environment conditions: reduced pressure $\left(\mathrm{a}: \mathrm{P}=0.5 \mathrm{Bar}, \mathrm{T}=24^{\circ} \mathrm{C}\right)$, reduced temperature $(\mathrm{b}$ : $\mathrm{P}=1 \mathrm{bar}, \mathrm{T}=-12{ }^{\circ} \mathrm{C}$ ) and simulated flight conditions with a reduced pressure and temperature $\left(\mathrm{c}: \mathrm{P}=0.5\right.$ Bar, $\left.\mathrm{T}=-12^{\circ} \mathrm{C}\right)[19]$.

The surface free energy was calculated based on Owens - Wendt model taking into account of the thermodynamic parameters under simulated flight conditions with reduced pressure and temperature $[18,24]$. Table 2 shows the surface free energy and its components of PDMS / silica coatings comparing with commercial coatings [18]. It can be seen that the total surface energy, dispersion component and polar component were reduced by $65.3 \%, 45.7 \%$ and $97.6 \%$ respectively compared with the commercial icephobic coatings. It is believed that the polar component mainly influences the adhesion between the water droplet and surface [18]. Therefore, the significantly reduced polar component implies a reduction of the adhesion of water droplet on the surface which facilitates water droplets rolling off and minimize icing accumulation. 
Table 2: Surface free energy and its components of PDMS/Silica coatings and commercial icephobic coatings measured in simulated flight conditions [18, 19]

\begin{tabular}{|c|c|c|c|}
\hline \hline & $\begin{array}{c}\text { Total } \\
\text { surface energy } \\
\left(\mathrm{mJ} / \mathrm{m}^{2}\right)\end{array}$ & $\begin{array}{c}\text { Dispersion } \\
\text { component } \\
\left(\mathrm{mJ} / \mathrm{m}^{2}\right)\end{array}$ & $\begin{array}{c}\text { Polar component } \\
\left(\mathrm{mJ} / \mathrm{m}^{2}\right)\end{array}$ \\
\hline $\begin{array}{c}\text { PDMS/silica } \\
\text { coatings }\end{array}$ & 25.7 & 24.7 & 0.7 \\
\hline $\begin{array}{c}\text { Commercial } \\
\text { coatings }\end{array}$ & 74.1 & 45.5 & 28.6 \\
\hline Reduction $(\%)$ & $65.3 \%$ & $45.7 \%$ & $97.6 \%$ \\
\hline \hline
\end{tabular}

To quantify the effect on ice adhesion strength by different pressures and temperatures, the work of adhesion, W, was calculated according to Young-Dupré equation [5]:

$$
\mathrm{W}=\sigma(1+\cos \theta)
$$

where $\sigma$ stands for the surface tension and $\theta$ stands for the water contact angle. According to Equation (3), the estimated work of adhesion between water and the PDMS/silica coatings measured in reduced pressure and temperature is 6 to 9 times of that measured in normal conditions. It could explain the higher ice adhesion strength of F-PDMS/silica coatings.

\subsection{Durability under water impinging erosion}

When an aircraft flies through the atmosphere, its surfaces and particularly its leading edge of wings may undergo impact by hydrometeors such as rain, hail and ice which can adversely affect the flight [39]. Similarly, as the skin of the aircraft, icephobic coatings will also encounter the same problem. Durability performance under water impinging erosion for the icephobic coatings is extremely important for the practical applications. Water impinging erosion test method was established and used to evaluate the durability of F-PDMS and F-PDMS/silica coatings. Water contact angle was measured before and after the erosion test. Wettability test results show water contact angle of $125^{\circ}$ before erosion test and $127^{\circ}$ after the erosion test for 
90 minutes for F-PDMS based coating. For the F-PDMS/silica coatings, WCA slightly reduced from $157^{\circ}$ to $151^{\circ}$ after the erosion.

To reveal the surface evolution during water impinging erosion, surface morphology characterization was performed for F-PDMS/silica coatings. Compared with the as-prepared surface (Figure 8(a)), the coatings after erosion test maintained similar surface morphology, as indicated in Figure 8(b). But a few big surfaces cracks formed and grew during the water impinging erosion. Cracks on these surfaces are more likely to occur and grow [40]. Therefore, although there was not too much change on the surface wettability with the specific water impinging erosion, the coating durability does need further improvement. Smooth surfaces fabricated by materials with better bonding properties might be a favourable direction for improvement of durability.

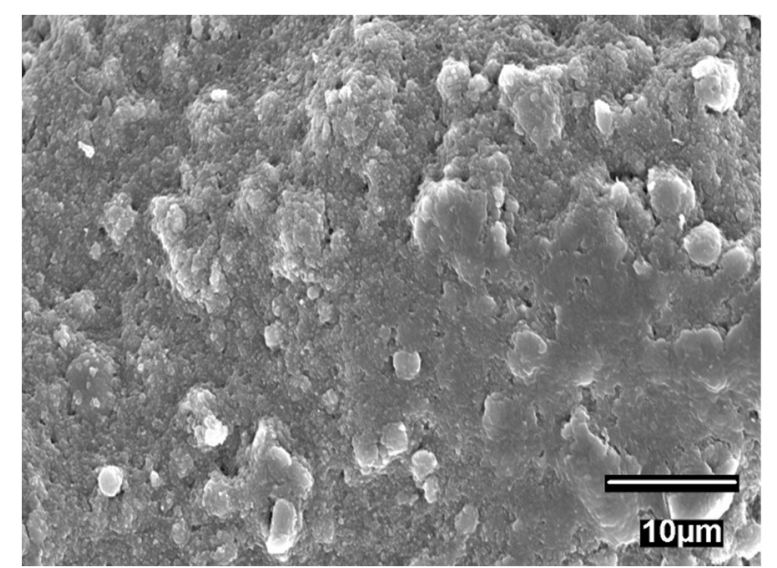

(a)

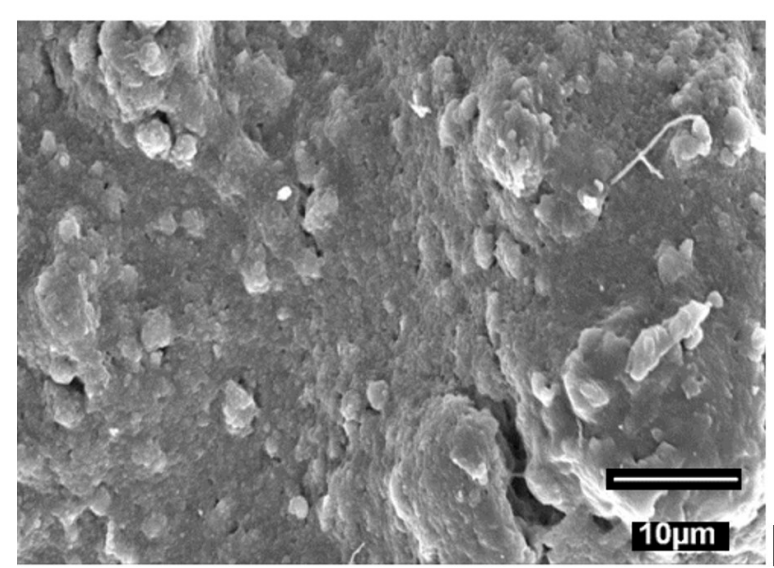

(b)

Figure 8 SEM images of F-PDMS/silica coating before erosion test (a) and after erosion test for 90 minutes (b).

\section{Conclusions}

Coatings based on fluorinated PDMS (F-PDMS) and fluorinated PDMS/silica (FPDMS/silica) nanoparticles have been developed aiming for ice protection for aircraft. 
Hydrophobicity test results in normal conditions with atmospheric pressure and room temperature show improved hydrophobicity by the fluorination of PDMS and the incorporation of silica nanoparticles. The water droplet icing test shows better anti-icing performance with F-PDMS/silica coatings due to better hydrophobicity. However, the ice adhesion test shows lower ice adhesion strength by fluorinated PDMS coatings without the addition of silica nanoparticles. The wetting behaviour measured under reduced pressure and temperature revealed much higher work of adhesion between water and F-PDMS/silica coatings under reduced pressure and/or temperature implying that higher surface free energy and roughnessinduced-hydrophobicity would play less function to the icephobicity especially for the de-icing performance in reduced pressure and temperature. A balance between the anti-icing and deicing performance needs to be reached when designing an icephobic coating. A compromise of the hydrophobicity by using smooth surface is critical to avoid the increase of mechanical bonding between the ice and the rough surface.

\section{Acknowledgement}

This project has received funding from the Clean Sky 2 Joint Undertaking under the European Union's Horizon 2020 research and innovation programme under grant agreement No CS2-AIR-GAM-2014-2015-O1. Cf. Art. 29.4 of [A2]. The authors also would like to thank the supports from Mr. Yan Zheng and Nanoscale \& Microscale Research Centre of The University of Nottingham.

\section{References}

[1] Federal Aviation Administration, The Icemaster Database and an Analysis of Aircraft Aerodynamic Icing Accidents and Incidents, 2015.

[2] Airbus Industrie, Getting to grips with cold weather operations a flight operations view, Blagnac France, 2000.

[3] A. Sulej, Ż. Polkowska, J. Namieśnik, Contaminants in airport runoff water in the vicinities of two international airports in Poland, Polish Journal of Environmental Studies 21 (2012) 725-739.

[4] A.J. Meuler, G.H. McKinley, R.E. Cohen, Exploiting topographical texture to impart icephobicity, ACS nano 4 (2010) 7048-7052. 
[5] A.J. Meuler, J.D. Smith, K.K. Varanasi, J.M. Mabry, G.H. McKinley, R.E. Cohen, Relationships between water wettability and ice adhesion, ACS applied materials \& interfaces 2 (2010) 3100-3110. [6] L. Zheng, Z. Li, S. Bourdo, K.R. Khedir, M.P. Asar, C.C. Ryerson, A.S. Biris, Exceptional superhydrophobicity and low velocity impact icephobicity of acetone-functionalized carbon nanotube films, Langmuir 27 (2011) 9936-9943.

[7] S. Jung, M. Dorrestijn, D. Raps, A. Das, C.M. Megaridis, D. Poulikakos, Are superhydrophobic surfaces best for icephobicity?, Langmuir 27 (2011) 3059-3066.

[8] S. Kulinich, S. Farhadi, K. Nose, X. Du, Superhydrophobic surfaces: are they really ice-repellent?, Langmuir 27 (2010) 25-29.

[9] V. Bahadur, L. Mishchenko, B. Hatton, J.A. Taylor, J. Aizenberg, T. Krupenkin, Predictive model for ice formation on superhydrophobic surfaces, Langmuir 27 (2011) 14143-14150.

[10] L. Cao, A.K. Jones, V.K. Sikka, J. Wu, D. Gao, Anti-icing superhydrophobic coatings, Langmuir 25 (2009) 12444-12448.

[11] S. Kulinich, M. Farzaneh, How wetting hysteresis influences ice adhesion strength on superhydrophobic surfaces, Langmuir 25 (2009) 8854-8856.

[12] A. Dotan, H. Dodiuk, C. Laforte, S. Kenig, The relationship between water wetting and ice adhesion, Journal of Adhesion Science and Technology 23 (2009) 1907-1915.

[13] R. Menini, Z. Ghalmi, M. Farzaneh, Highly resistant icephobic coatings on aluminum alloys, Cold Regions Science and Technology 65 (2011) 65-69.

[14] S. Farhadi, M. Farzaneh, S. Kulinich, Anti-icing performance of superhydrophobic surfaces, Applied Surface Science 257 (2011) 6264-6269.

[15] T.-S. Wong, S.H. Kang, S.K. Tang, E.J. Smythe, B.D. Hatton, A. Grinthal, J. Aizenberg, Bioinspired self-repairing slippery surfaces with pressure-stable omniphobicity, Nature 477 (2011) 443-447.

[16] S.H. Yang, M. Nosonovsky, H. Zhang, K.-H. Chung, Nanoscale water capillary bridges under deeply negative pressure, Chemical Physics Letters 451 (2008) 88-92.

[17] M. Nosonovsky, B. Bhushan, Phase behavior of capillary bridges: towards nanoscale water phase diagram, Physical Chemistry Chemical Physics 10 (2008) 2137-2144.

[18] L.C. L Mazzola, N. Felde, G. Bruno, B Galasso, V Quaranta, F Albano, A Auletta, Development and Technological Characterization of Multi-functional Aeronautical Coating From Lab-Scale to the Relevant Environment, Journal of Aeronautics \& Aerospace Engineering 6 (2017) 1.

[19] L. Mazzola, GAINS report: Surface Characterizations of new coatings developed by University of Nottingham, (2017).

[20] Q. Fu, X. Wu, D. Kumar, J.W. Ho, P.D. Kanhere, N. Srikanth, E. Liu, P. Wilson, Z. Chen, Development of sol-gel icephobic coatings: effect of surface roughness and surface energy, ACS applied materials \& interfaces 6 (2014) 20685-20692.

[21] H. Li, X. Li, C. Luo, Y. Zhao, X. Yuan, Icephobicity of polydimethylsiloxane-b-poly (fluorinated acrylate), Thin Solid Films 573 (2014) 67-73.

[22] J. Li, Y. Zhao, J. Hu, L. Shu, X. Shi, Anti-icing performance of a superhydrophobic PDMS/modified nano-silica hybrid coating for insulators, Journal of Adhesion Science and Technology 26 (2012) 665-679.

[23] Y. Wang, X. Yao, J. Chen, Z. He, J. Liu, Q. Li, J. Wang, L. Jiang, Organogel as durable anti-icing coatings, Science China Materials 58 (2015) 559-565.

[24] L. Mazzola, E. Bemporad, F. Carassiti, An easy way to measure surface free energy by drop shape analysis, Measurement 45 (2012) 317-324.

[25] J. Liu, Z.A. Janjua, M. Roe, F. Xu, B. Turnbull, K.-S. Choi, X. Hou, Super-hydrophobic/icephobic coatings based on silica nanoparticles modified by self-assembled monolayers, Nanomaterials 6

(2016) 232.

[26] C. Laforte, A. Beisswenger, Icephobic material centrifuge adhesion test, Proceedings of the 11th International Workshop on Atmospheric Icing of Structures, IWAIS, Montreal, QC, Canada, 2005, pp. $12-16$. 
[27] R. Menini, M. Farzaneh, Advanced icephobic coatings, Journal of adhesion science and technology 25 (2011) 971-992.

[28] M. Matsumoto, K.-i. Tanaka, R. Azumi, Y. Kondo, N. Yoshino, Template-Directed Patterning Using Phase-Separated Langmuir- Blodgett Films, Langmuir 20 (2004) 8728-8734.

[29] D. Sanli, C. Erkey, Effect of polymer molecular weight and deposition temperature on the properties of silica aerogel/hydroxy-terminated poly (dimethylsiloxane) nanocomposites prepared by reactive supercritical deposition, The Journal of Supercritical Fluids 105 (2015) 99-107.

[30] B. Bhushan, M. Nosonovsky, The rose petal effect and the modes of superhydrophobicity, Philosophical Transactions of the Royal Society of London A: Mathematical, Physical and Engineering Sciences 368 (2010) 4713-4728.

[31] A. Giacomello, S. Meloni, M. Chinappi, C.M. Casciola, Cassie-Baxter and Wenzel states on a nanostructured surface: phase diagram, metastabilities, and transition mechanism by atomistic free energy calculations, Langmuir 28 (2012) 10764-10772.

[32] A. Peters, C. Pirat, M. Sbragaglia, B. Borkent, M. Wessling, D. Lohse, R.G. Lammertink, CassieBaxter to Wenzel state wetting transition: Scaling of the front velocity, The European Physical Journal E 29 (2009) 391-397.

[33] M.J. Kreder, J. Alvarenga, P. Kim, J. Aizenberg, Design of anti-icing surfaces: smooth, textured or slippery?, Nature Reviews Materials 1 (2016) 15003.

[34] O. Kylián, M. Petr, A. Serov, P. Solař, O. Polonskyi, J. Hanuš, A. Choukourov, H. Biederman, Hydrophobic and super-hydrophobic coatings based on nanoparticles overcoated by fluorocarbon plasma polymer, Vacuum 100 (2014) 57-60.

[35] Y. Shen, J. Tao, H. Tao, S. Chen, L. Pan, T. Wang, Anti-icing potential of superhydrophobic Ti6Al4V surfaces: Ice nucleation and growth, Langmuir 31 (2015) 10799-10806.

[36] V. Hejazi, K. Sobolev, M. Nosonovsky, From superhydrophobicity to icephobicity: forces and interaction analysis, Scientific reports 3 (2013).

[37] P. Eberle, M.K. Tiwari, T. Maitra, D. Poulikakos, Rational nanostructuring of surfaces for extraordinary icephobicity, Nanoscale 6 (2014) 4874-4881.

[38] L. Mazzola, Aeronautical livery coating with icephobic property, Surface Engineering 32 (2016) 733-744.

[39] O. Gohardani, Impact of erosion testing aspects on current and future flight conditions, Progress in Aerospace Sciences 47 (2011) 280-303.

[40] R. Zhang, P. Hao, F. He, Rapid Bouncing of High-Speed Drops on Hydrophobic Surfaces with Microcavities, Langmuir 32 (2016) 9967-9974. 